\title{
STATIC AND DYNAMIC BRIDGE AMPLIFIER CALIBRATION ACCORDING TO ISO 4965-2
}

\author{
L. Klaus ${ }^{1}$ \\ ${ }^{1}$ Physikalisch-Technische Bundesanstalt (PTB), Bundesallee 100, 38116 Braunschweig, Germany, leonard.klaus@ptb.de
}

\begin{abstract}
:
Dynamic measurements to correctly adjust the magnitude in fatigue testing require a dynamic calibration of force transducers and conditioning electronics if the dynamic loads are to be measured correctly. International standard ISO 4965 describes a calibration method for these components. At PTB, such a calibration of bridge amplifiers has now been performed for the first time. The calibration includes tests with static and dynamic signals. The reference for the calibration is the dynamic bridge standard of PTB. It is particularly suitable for this application as it can generate static and dynamic signals, and thus all investigations can be performed with one reference and in a reasonably short time. The signal creation and the data analysis were carried out using the open source program GNU Octave. For the data analysis, a semi-automatic procedure was developed to simplify the process. Calibrations of two digital bridge amplifiers were carried out.
\end{abstract}

Keywords: dynamic calibration; dynamic bridge amplifier calibration; fatigue testing

\section{INTRODUCTION}

In fatigue testing, the cyclic loading and unloading of a specimen is performed. The load itself can be measured by a force transducer which needs to be calibrated dynamically. This could be carried out using a replica specimen with applied strain gauges (a so-called dynamic calibration device, DCD) as described as one option in international standard ISO 4965-1 (2012) [1]. In order to be able to determine the correct force amplitude, the signal conditioning electronics for the strain gauges of the DCD need to be calibrated as well. The calibration procedure is described in part 2 of the aforementioned standard: ISO 4965-2 (2012) [2].

The standard describes an assessment not only of the conditioning amplifier, but also of the subsequent data acquisition electronics and the possible data display. This measurement procedure (described as a calibration in the standard) is rather a conformity assessment, which defines the maximum deviations

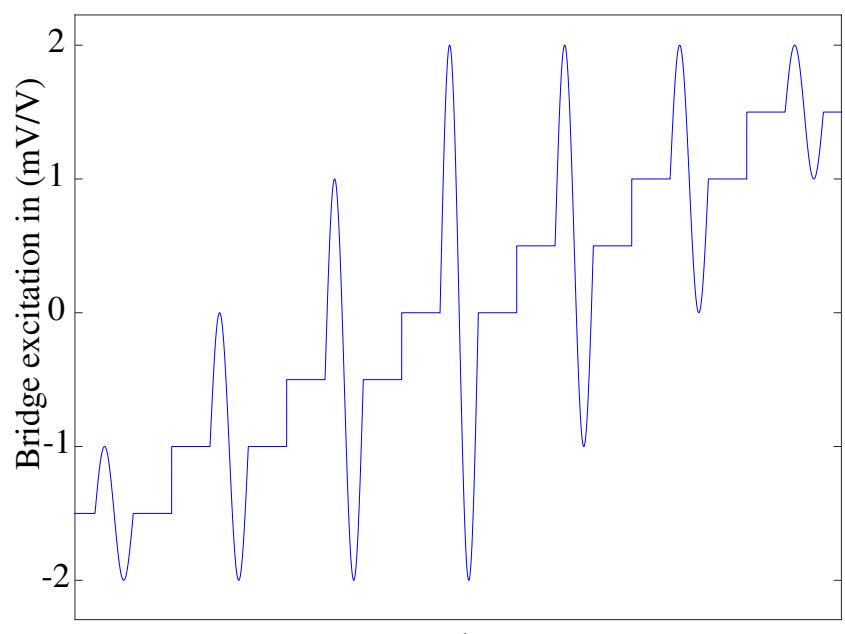

Time

Figure 1: Dynamic excitation waveform according to ISO 4965-2 (2012).

which must not be exceeded in order to pass the assessment.

The assessment consists of three separate tests:

Static excitation Nine different static excitation levels in steps of $0.5 \mathrm{mV} / \mathrm{V}$ from $-2 \mathrm{mV} / \mathrm{V}$ to $2 \mathrm{mV} / \mathrm{V}$ are generated, and the derived values are to be compared with the excitation.

Dynamic excitation A set of nine sinusoids of common frequency with different offset values and amplitudes are generated. The generated waveform is depicted in Figure 11. For each sinusoid, the maximum and the minimum values are determined and compared with the reference.

\section{Dynamic excitation with superimposed distortions}

The same set of sinusoids as used for the dynamic excitation is superimposed with higher frequency harmonic distortions. The total harmonic distortions are to be $0.125 \% \pm 0.01 \%$.

The standard document is rather strict in terms of excitation levels, and it always requires $2 \mathrm{mV} / \mathrm{V}$ rather than levels adjustable to the application. This is unexpected, as the occurring bridge excitation levels of self-applied strain gauges can differ significantly, based on the strain gauges, the mechanical parameters of the test pieces, and the placement of the strain gauges on those test pieces. 
On the other hand, for the superimposed harmonic distortions, only one parameter is defined: the overall relative magnitude. In contrast, for the frequencies, the number of frequencies and the distribution of the magnitude of the harmonic distortions within these different frequencies are not defined at all.

\section{BRIDGE REFERENCE}

The requirements for the static assessment of the conditioning electronics can be fulfilled by any static bridge standard with sufficient precision. However, the dynamic tests are quite demanding, as dynamic and static components need to be superimposed in order to generate the required waveforms.

PTB developed a dynamic bridge standard [3] which can generate static as well as dynamic bridge excitation to frequencies of up to $10 \mathrm{kHz}$. It is already incorporated in a calibration set-up for the dynamic calibration of bridge amplifiers using sinusoidal excitation signals [4]. This bridge reference behaves similarly to a strain gauge transducer equipped with a full Wheatstone bridge circuit with a resistance of $350 \Omega$. The signal generation is based on two multiplying digital-analogue converters (MDACs) providing a strictly ratiometric and groundindependent (floating) output based on the supply voltage provided by the bridge amplifier. The bridge standard can generate arbitrary waveforms and therefore directly generate all the necessary waveforms required in ISO 4965-2.

The bridge standard is controlled by a computer using a fibre link to avoid ground loops. LabView software is used to set the operation modes and to transfer the waveforms to the bridge standard.

\section{CALIBRATION SET-UP AND OPERATION}

An assessment of the conditioning electronics of the DCD according to ISO 4965-2 always incorporates not only the bridge amplifier, but also the successively connected signal conditioning and acquisition electronics, filtering, and (possibly) the data display. We therefore decided on a calibration set-up, in which the signal generation and the signal analysis are split up (see Figure 2). Such a set-up is possible, because ISO 4965-2 only takes into account magnitudes but not the timing, e.g. phase delays of the devices under test, which seems reasonable for this application. The dynamic bridge standard generates the reference signals for the three different tests. The dynamic signals are generated in a loop. The customer's signal conditioning electronics

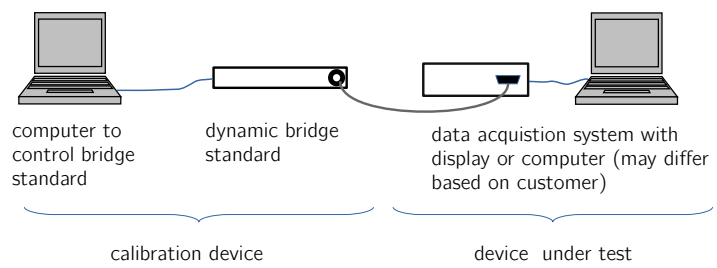

Figure 2: Design of the calibration set-up with the reference (dynamic bridge standard, left) and the signal conditioning and data acquisition system with display or computer (may differ based on customer, right).

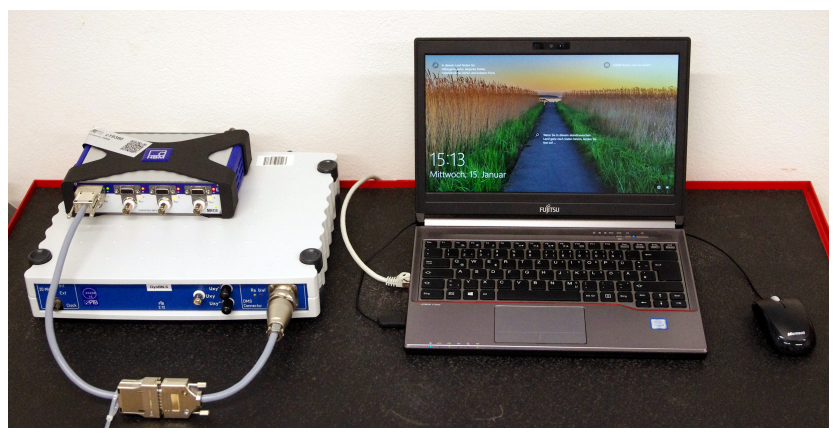

Figure 3: Calibration set-up consisting of a digital bridge amplifier under test (top left) connected to the dynamic bridge standard (bottom left) and via Ethernet to the computer to acquire the data (right). The computer used to program the bridge standard is not shown in the picture.

(i.e. the bridge amplifier) and the data acquisition electronics will be connected to the bridge standard and operated under the same conditions as in a fatigue test. These conditions include input span, filter settings, sampling rates, operating and data acquisition software (including the same software version), and the interfaces used in the case of a digital data transmission. A typical set-up with the bridge standard, a digital bridge amplifier, and a computer to save the data is depicted in Figure 3

The data is then acquired by the customer's system and saved for the data analysis. To be independent of the data acquisition system, the data analysis is carried out on a PTB computer, and the data is transferred using common file formats. In the case of incompatible file formats of the customer's system and PTB's data analysis software, data conversion would be necessary.

\section{DATA GENERATION}

The generation of the required waveforms for the dynamic tests was carried out using GNU Octave, the open source numerical computation software [5]. These waveforms were saved in a text file and then processed by LabView software to transfer the data to the bridge standard. The dynamic bridge standard can be operated in a static and a dynamic operation mode. The operation mode is set in the LabView software. In the latter, the transferred waveforms are generated in a loop. 
As the bridge standard can provide arbitrary waveforms, the required sinusoids for the dynamic testing (cf. Figure 1) can be generated directly. Based on one waveform file, several different excitation frequencies can be produced by altering the sampling frequency of the MDACs of the bridge standard (and therefore altering how "fast" the waveform is processed). The sampling frequency was chosen to be at least twenty times higher than the excitation frequency to preserve a sufficiently densely sampled waveform.

As previously mentioned, the superimposed harmonic distortions are only defined in their relative level of $0.125 \% \pm 0.01 \%$, but not in their frequency content or in the distribution between different multiples of the excitation frequency. As we are dealing with amplifiers measuring mechanical oscillations, for the described set-up, only the typically most dominant third and fifth harmonic were chosen to generate the required total harmonic distortions. Two different popular definitions of the total harmonic distortions exist: one which refers to the waveform's magnitude and one which compares it with the waveform's RMS value [6]. The corresponding levels of the harmonics were chosen based on the former definition, following the recommendation in [6] giving

$\mathrm{THD}=\frac{\sqrt{\sum_{n=2}^{\infty} A_{n}^{2}}}{A_{1}^{2}}$

with the amplitudes being $A_{1}$ for the fundamental sinusoid and the amplitudes of its different harmonics being $A_{n}$. For the desired $0.125 \%$ total harmonic distortions, values of $0.122 \%$ of the third harmonic and $0.0272 \%$ of the fifth harmonic were chosen to achieve the desired level of harmonic distortions according to ISO 4965-2.

\section{DATA ANALYSIS}

For the data analysis, the derived output values of the signal conditioning and acquisition chain have to be compared with the nominal values. The standard defines intervals within which the output value has to be.

For the static tests, the nominal values are known, while for the dynamic tests, the maximum and minimum values are compared. These values are well known for the sinusoidal excitation without distortions and can be derived directly from the waveform file for the test with superimposed harmonic distortions.

As the calibration according to ISO 49652 is actually a conformity assessment with the given conformity intervals, the recommendations in the JCGM guide on measurement uncertainty in conformity assessment [7] were taken into account. This means, for the conformity interval, that the expanded measurement uncertainty of the generated values (i.e. the measurement uncertainty of the dynamic bridge standard) needs to be subtracted from the interval, narrowing it accordingly. For simplicity, one measurement uncertainty covering all three tests was estimated. The relative expanded measurement uncertainty (coverage interval $95 \%$ ) is $0.1 \%$ at a nominal excitation level of $2 \mathrm{mV} / \mathrm{V}$.

For the static measurements, the standard requires an analysis of three samples at seven given excitation levels. All samples have to be within the conformity interval of $\pm 0.01 \mathrm{mV} / \mathrm{V}$ or $\pm 0.5 \%$ related to the nominal excitation level of $2 \mathrm{mV} / \mathrm{V}$.

The dynamic excitations with undisturbed sinusoidal excitation and with superimposed distortions are carried out with seven sine waves of different amplitudes and offsets but with a common frequency, as depicted in Figure 1 . For the conformity assessment, the standard requires all maximum and minimum values of the seven sine waves to be within the specified conformity interval. These values are derived directly from the measurement data without applying a curve fit or similar algorithms, and they therefore take into account all noise present in the measured waveform. The conformity intervals need to be within $\pm 0.5 \%$, similar to the requirements for the static tests. The dynamic excitations with undisturbed sinusoidal excitation and with superimposed distortions are carried out with seven sine waves of different amplitudes and offsets but with a common frequency, as depicted in Figure 11. For the conformity assessment, the standard requires all maximum and minimum values of the seven sine waves to be within the specified conformity interval. These values are derived directly from the measurement data without applying a curve fit or similar algorithms, and they therefore take into account all noise present in the measured waveform. The conformity intervals need to be within $\pm 0.5 \%$, similar to the requirements for the static tests.

The data analysis was carried out using GNU Octave as well. For the static measurement, a set of data points was loaded into Octave and compared with the nominal value. For the dynamic tests, it was necessary to identify the different sine waves to distinguish the maximum and minimum values. Based on the transfer function of the measuring chain, lowpass filters can make automatic wave identification impossible. Therefore, a semi-automatic approach was chosen. The operator selects the start and 
end of each sine wave by clicking on them with a cursor marker, and the data processing is carried out automatically afterwards. The results are plotted with the conformity interval and the highlighted maximum and minimum values are coloured in green if the result complies with the requirements, or in red if the test failed for the displayed waveform. An example is depicted in Figure 4.

\section{RESULTS}

The first measurements were carried out by evaluating digital measuring amplifiers from HBM's QuantumX MX4xx range! These universal amplifiers condition bridge (and other) inputs and digitise the signals afterwards. The digital data is transferred via Ethernet or Firewire to a computer. The bridge amplifiers can be operated with a carrier frequency or with DC bridge excitation.

While the carrier-frequency operation mode has advantages in terms of noise levels, the dynamic properties of DC amplifiers are typically better. Therefore, carrier-frequency amplifiers are used rather rarely for dynamic applications. If carrier-frequency amplifiers are used in such dynamic applications, one should take care to limit the frequencies of interest in the measurement signal to not more the $1 / 5$ to $1 / 10$ of the carrier frequency (depending on the specifications of the amplifier). Furthermore, ISO 4965-2 [2, Section 2.2] advises only the use of a DC voltage as a supply voltage of the bridge circuit.

The analysed amplifiers (one of them depicted in Figure (3) were calibrated at $5 \mathrm{~Hz}, 20 \mathrm{~Hz}, 50 \mathrm{~Hz}$, and $100 \mathrm{~Hz}$. The frequency range chosen was based on typical frequencies in fatigue testing. A section of 4 out of 7 sine waves is depicted in Figure 4 as an example at a frequency of $100 \mathrm{~Hz}$. The sampling rate used for the data acquisition in these tests was $4800 \mathrm{~S} / \mathrm{s}$.

For the calibration, the data was acquired using HBM Catman, a data acquisition and processing software tool. The data was then saved as a binary file in a Matlab-compatible format (.mat) which was imported into Octave for the data processing.

The result of the conformity assessment showed that both analysed amplifiers successfully passed the requirements of ISO 4965-2 for all three tests in the frequency range of up to $100 \mathrm{~Hz}$.

\footnotetext{
${ }^{1}$ Commercial devices are identified in this paper only to adequately specify the devices under test. Such identification does not imply recommendation by PTB, nor does it imply that the equipment identified is necessarily the best available for the purpose.
}
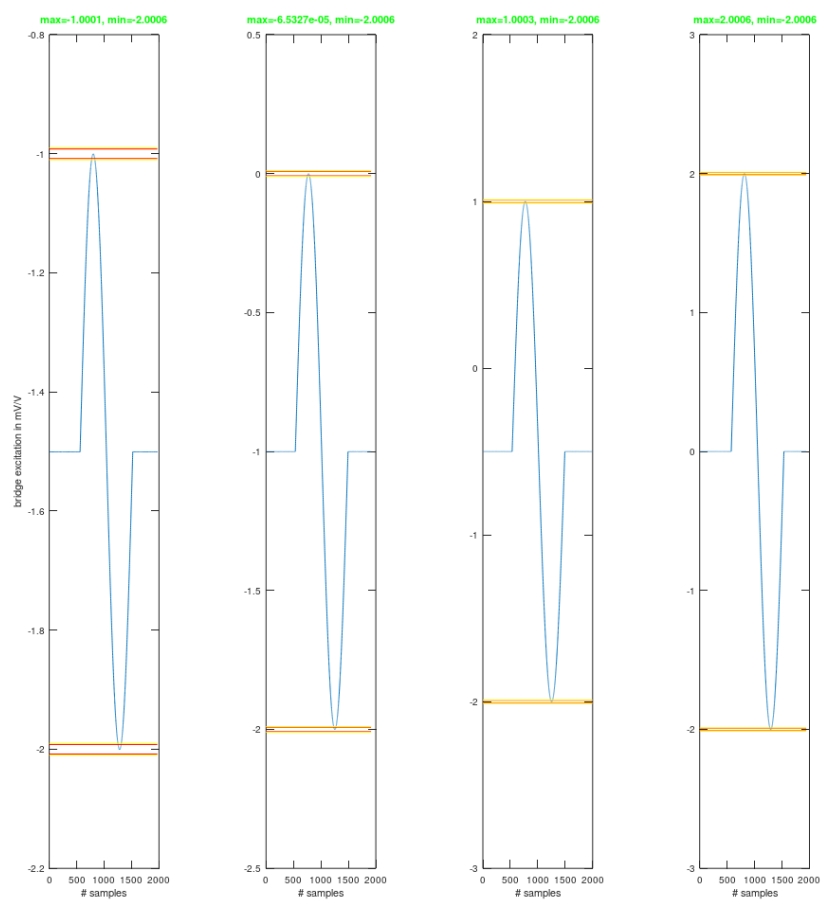

Figure 4: Example of the data analysis output: screen shot of the data analysis software for four of seven waveforms with undisturbed sinusoidal excitation at a frequency of $100 \mathrm{~Hz}$.

\section{SUMMARY AND OUTLOOK}

A new calibration set-up for the conformity assessment of signal conditioning electronics and data acquisition hardware used for fatigue tests is presented. The conformity assessment was carried out according to ISO 4965-2 and incorporates three different tests with static and dynamic signals. The data was acquired under the same settings as in the later application, and the output was subsequently analysed independently with GNU Octave.

Results from two analysed digital amplifiers successfully prove the compliance of the analysed amplifiers to ISO 4965-2 in the frequency range from $5 \mathrm{~Hz}$ to $100 \mathrm{~Hz}$ and in the static regime.

Currently, PTB is preparing to offer any interested customer these conformity assessments as a service in the frequency range from $1 \mathrm{~Hz}$ to $2000 \mathrm{~Hz}$.

\section{ACKNOWLEDGMENTS}

This work is part of the Joint Research Project 18SIB08 Comprehensive Traceability for Force Metrology Services (ComTraForce) of the European Metrology Programme for Innovation and Research (EMPIR). The EMPIR is jointly funded by the EMPIR participating countries within EURAMET and the European Union. 


\section{REFERENCES}

[1] ISO/TC 164/SC 5, "ISO 4965-1:2012, Metallic Materials-Dynamic Force Calibration for Uniaxial Fatigue Testing-Part 1: Testing Systems", International Organization for Standardization, Geneva, Switzerland, 2012.

[2] ISO/TC 164/SC 5, "ISO 4965-2:2012, Metallic Materials-Dynamic Force Calibration for Uni-axial Fatigue Testing-Part 2: Dynamic Calibration Device (DCD) Instrumentation", International Organization for Standardization, Geneva, Switzerland, 2012.

[3] M. Florian Beug, Harald Moser, and Günther Ramm, "Dynamic Bridge Standard for Strain Gauge Bridge Amplifier Calibration", Proc. of 2012 Conference on Precision Electromagnetic Measurements (CPEM), pp. 568-69, Washington D.C., USA, 2012. DOI: 10.1109/CPEM.2012.6251056

[4] Leonard Klaus, M. Florian Beug, and Thomas Bruns, "Setup for the Dynamic Calibration of Bridge Amplifiers from DC up to $10 \mathrm{kHz}$ ", Acta IMEKO, vol. 8 (1), pp. 19-24, March 2018. DOI: 10.21014/acta_imeko.v8i1.657

[5] Free Software Foundation, Inc., "GNU Octave", https://www .octave.org

[6] Doron Shmilovitz, "On the Definition of Total Harmonic Distortion and Its Effect on Measurement Interpretation", IEEE Transactions on Power Delivery, vol. 20 (1), pp. 526-28, January 2005. DOI: 10.1109/TPWRD.2004.839744

[7] Joint Committee for Guides in Metrology, "JCGM 106:2012, Evaluation of Measurement Data-The Role of Measurement Uncertainty in Conformity Assessment", Bureau International des Poids et Mesures, Sèvres, France 2012. 